\title{
Laser-induced cratering of a 3DCC material at mesoscale: Experiments and simulations
}

\author{
Vincent Jaulin ${ }^{1, *}$, David Hébert $^{1}$, Bertrand Aubert ${ }^{1}$, Jean-Luc Rullier $^{1}$, Frédéric Malaise ${ }^{1}$, and Emilien Lescoute $^{2}$ \\ ${ }^{1}$ CEA CESTA, 15 avenue des Sablières, CS60001, 33116 Le Barp Cedex, France \\ ${ }^{2}$ CEA DIF, Bruyères-le-Châtel, 91297 Arpajon Cedex, France
}

\begin{abstract}
This study concerns the damaging of a 3-Dimension Carbon/Carbon (3DCC) composite material under dynamic loading. Experiments were performed with a laser facility delivering energies between 13 and $40 \mathrm{~J}$ with 100 nanoseconds pulse duration. The focal spot diameter was $250 \mu \mathrm{m}$, leading to dynamic pressure up to $10 \mathrm{GPa}$. The focal spot being smaller than the size of the composite components, it allows us to study the dynamic behaviour of the material at mesoscopic scale. The dynamic process of this cratering is then investigated via 3D numerical simulations, and a Johnson-Holmquist model is proposed. It appears that comparison of simulations with experimental results is useful to identify the dynamic strength of individual components of composite materials.
\end{abstract}

\section{Introduction}

The dynamic behaviour of carbon-based composite materials under high velocity impacts is an active field of research. Both experimental [1][2] and numerical results [3][4] emphasize the major role played by the mesostructure. However, mesoscale modelling requires much material data about damaging and cratering processes of elementary components (graphite matrix and fibers). The purpose of this paper is to provide some insight on the damaging processes in a 3DCC composite. The first section describes experimental results. A mesoscopic model, based on the Johnson-Holmquist model, is proposed in the second section with a complete set of parameters. Comparison of simulations with experiments is presented in the third section.

\section{Experiments}

\subsection{Material}

The material under study is a 3-Dimension Carbon/Carbon composite [3]. Its structure is based on a three directionnal orthogonal weaving of carbon fibers embedded in a carbon matrix. The fibers, called X, Y and Z-fibers, correspond to yarn bundle oriented in the corresponding cartesian directions (Fig. 1). The carbon matrix, partially porous, completes free spaces and interfaces resulting in a global composite density of $1950 \mathrm{~kg} / \mathrm{m}^{3}$. Observation of 3DCC with profilometry highligths the structure of the composite surface. We notice that emerged mesoconstituants at the surface are varying with the location on the material (Fig. 2).

\footnotetext{
*e-mail: vincent.jaulin@ cea.fr
}

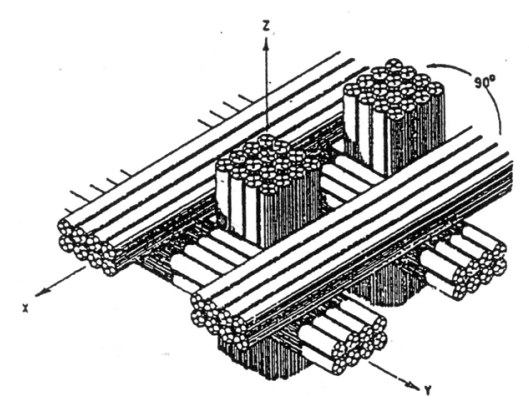

Fig. 1. 3D orthogonal weave.

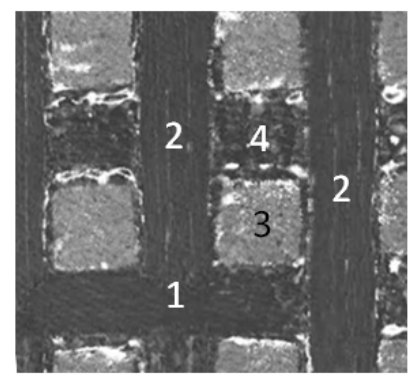

1) X-fiber

2) Y-fiber

3) Z-fiber

4) Matrix

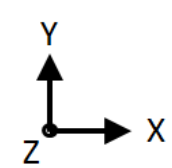

Fig. 2. 3DCC profilometer observation.

\subsection{Laser shocks}

Cratering experiments have been conducted using GCLT laser facility [5]. Irradiation of a target with a short and intense laser pulse generates a high pressure plasma which, when expanding, initiates a shockwave in the material behind. The laser delivers energies between 13 and $40 \mathrm{~J}$ with $100 \mathrm{~ns}$ pulse duration at $1057 \mathrm{~nm}$ wavelength. The focal 
spot diameter was $250 \mu \mathrm{m}$, which is smaller than the dimension of the fiber section. The laser precision allowed us to target a component in particular. In this study, the loading was concentrated on Z-fibers of which filaments are directed in the shock direction. Post-experiment observation of craters with an interferometric profilometer were performed systematically for all craters (Fig. 3).
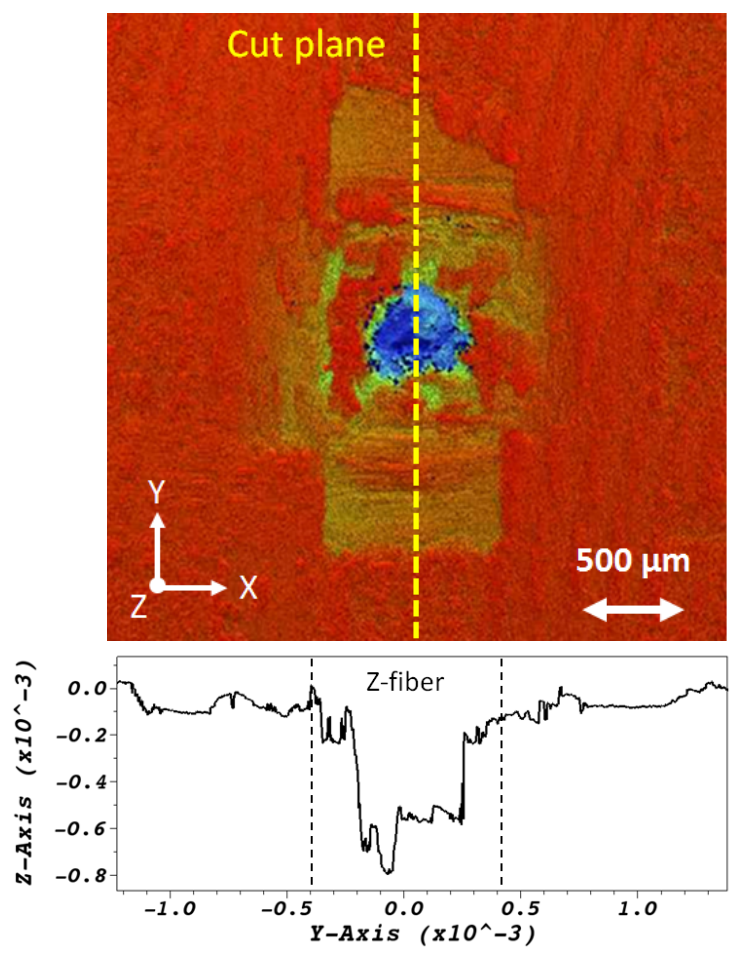

Fig. 3. a- Crater observation by profilometry -b- Crater profile at the cut plane. Laser configuration : $\varnothing=250 \mu m, \Delta t=100$ $n s, E=36.25 \mathrm{~J}$.

Main observations, based on 18 laser-induced craters with energies from $13 \mathrm{~J}$ to $36 \mathrm{~J}$, are listed below :

1. Craters for same laser energy are overall similar which goes along the idea of laser impact reproductibility ;

2. Characteristic dimensions as depth, diameter and volume of craters increase continuously with the level of energy delivered by the laser;

3. Contrary to an isotropic material, damage is linked with the structure of the composite. Sides of the craters regularly follow interfaces between the components for the strongest shots (Fig. 3-a);

4. Differences in the sides of the crater are noted depending if the component adjacent to the targeted Z-fiber is matrix or fibers and its predamage state. Fibers can be partially or totally torn off. Several cases highligthed disbondment of the fiber whithout removal.
5. The bottom of the crater is uneven particularly for the most energetic shots (Fig. 3-b). Several discontinuities are noticed which contrasts with the smooth bottom observed with same configuration laser impact on an isotropic carbon material [5][6]. Anisotropy of the fiber and predamage may cause this phenomenon as damage tends to follow interfaces filament/bond and weaknesses.

These observations lead us to assume damaged material is not entirely pulverised but ejected by blocks.

\section{Modelling}

\subsection{Material model}

We use the Eulerian hydrocode Hesione developped at CEA. A 3-Dimension mesoscale model was considered with constitutive laws specific to each components. Simplifications were realized by considering the components as isotropic material. Although the material is the same, different constitutive laws were considered according to the orientation of the filaments with the shock direction. At each time step, Hesione solves the three conservations equations (mass, energy, momentum) with the stress tensor $\overline{\bar{\sigma}}$ split in two parts :

$$
\overline{\bar{\sigma}}=-P \overline{\bar{I}}+\overline{\bar{S}}
$$

with $\mathrm{P}$ the hydrostatic pressure, $\overline{\bar{I}}$ the identity matrix and $\overline{\bar{S}}$ the deviatoric stress tensor.

The constitutive law used in this model is similar to the Johnson Holmquist (JH-2) model [7]. JH-2 model is applicable for brittle materials subjected to large strains, high strain rates and high pressures. It was formulated with a polynomial equation of state which depends on the energy (Eq. 2) :

$P=\left\{\begin{array}{lll}K_{1} \cdot \mu+K_{2} \cdot \mu^{2}+K_{3} \cdot \mu^{3}\left(1-\frac{\Gamma}{2} \mu\right)+\Gamma \cdot \rho \cdot E & \text { if } \quad \mu>0 \\ K_{1} \cdot \mu\left(1-\frac{\Gamma}{2} \mu\right)+\Gamma \cdot \rho \cdot E & \text { if } \quad \mu<0\end{array}\right.$

where $K_{1}, K_{2}, K_{3}$ are material constants obtained from fits to plates impact experiments [3] and respecting initial material density, $\Gamma$ is the Grüneïsen coefficient, $\rho$ the density and $\mu$ the contracted coefficient $\left(\mu=\rho / \rho_{0}-1\right)$. Table 1 details the coefficients for each components. The equation of state of those components are similar for high pressure as the materials are supposed equivalent to dense graphite. Due to its porosity, matrix EOS is slightly different at low pressure as its porosity entails a compaction phasis before reaching the dense behaviour. 
Table 1. EOS and elasticity coefficients for all components.

\begin{tabular}{l|ccc}
\hline Component & Z-fiber & X\&Y-fiber & Matrix \\
\hline$\rho_{0}\left(\mathrm{~kg} / \mathrm{m}^{3}\right)$ & 2041 & 2041 & 1760 \\
$K_{1}(\mathrm{GPa})$ & 12.4 & 8.16 & 2.3 \\
$K_{2}(\mathrm{GPa})$ & 0 & 0 & -25 \\
$K_{3}(\mathrm{GPa})$ & 250 & 280 & 120 \\
$\Gamma_{0}(-)$ & 0.8 & 0.8 & 0.8 \\
$\mathrm{G}(\mathrm{GPa})$ & 335 & 20 & 6.58 \\
\hline
\end{tabular}

In the $\mathrm{JH} 2$ model, the deviatoric stress tensor is limited by a Von Mises criterium. The yield stress is a damage D $\in[0 ; 1]$ based interpolated function of intact and fractured strength $\sigma_{i}^{*}$ and $\sigma_{f}^{*}$ respectively (Eq. 3). The intact and fractured strength are represented by Eq. 4 :

$$
\begin{gathered}
\sigma^{*}=\sigma_{i}^{*} \cdot(1-D)+\sigma_{f}^{*} \cdot D \\
\text { with }\left\{\begin{array}{l}
\sigma_{i}^{*}=A\left(P^{*}+T^{*}\right)^{N}(1+C \ln \dot{\varepsilon}) \\
\sigma_{f}^{*}=B\left(P^{*}\right)^{M}(1+C \ln \dot{\varepsilon}) \\
\sigma_{f}^{*}<\sigma_{i}^{*}
\end{array}\right.
\end{gathered}
$$

where A, B, C, M, N are parameters. The normalized stresses $\left(\sigma^{*}, \sigma_{i}^{*}, \sigma_{f}^{*}\right)$ have the following form, $\sigma / \sigma_{H E L}$, with $\sigma$ the actual equivalent stresses and $\sigma_{H E L}$ the equivalent stress at the Hugoniot Elastic Limit (HEL), $P^{*}$ the normalized hydrostatic pressure $\left(P / P_{H E L}\right)$ and $T^{*}$ the normalized maximum tensile hydrostatic pressure $\left(T / P_{H E L}\right)$.

Intact material coefficients $(\mathrm{A}, \mathrm{N})$ and the HEL of all components were deduced from plate impact experimental results [3] (not shown here). Assuming little strain rate depandancy, we chose $\mathrm{C}=0$ and consider transition from $D=0$ to $D=1$ as instantaneous once a damage criterium is reached. Supposing all the components have same behaviour once fragmented, equivalent stresses for the damaged components are the same. As it seems unphysical that fragmented equivalent stress surpass the intact one, limitation of the fragmented criteria by the intact one was adopted. In this model, two damage criteria are considered. In compression, damage happens once the plastic strain limit is exceeded which impacts the mechanical properties of the material by setting the damage coefficient $\mathrm{D}$ as 1 . In tension, the material is damaged once the maximum tensile pressure $\mathrm{T}$ is exceeded which entails same treatment but also relaxation of pressure and stresses to zero. Tensile damage tresholds $\mathrm{T}$ for the matrix and the fibers were supposed equal to the quasistatic tensile limit of the $3 \mathrm{DCC}$ in the directions $\mathrm{X}$ and $\mathrm{Z}$ respectively [3]. Stress equivalent limit for the damaged material $\sigma_{f}^{*}$ and plastic strain limit were calibrated (Tab. 2) based on the laser induced cratering results.
Table 2. Plasticity/damage coefficients of the JH2 model.

\begin{tabular}{l|ccc}
\hline Component & Z-fiber & X\&Y-fiber & Matrix \\
\hline A & 0.0932 & 0.315 & 0.195 \\
B & 0.00259 & 0.0288 & 0.0229 \\
C & 0 & 0 & 0 \\
M & 1 & 1 & 1 \\
N & 0.6 & 0.6 & 0.6 \\
T $(\mathrm{MPa})$ & 276 & 276 & 195 \\
LEH (MPa) & 200 & 200 & 70 \\
Failure strain $(\%)$ & 8 & 10 & 20 \\
\hline
\end{tabular}

\subsection{Laser sollicitation}

Interactions between laser and the material are not dealt with in the Hesione code. A study using the Finite Element code Esther [5][8] determined the profile of pressure applied as a boundary condition in Hesione. This profile of pressure, called ablation pressure, is dependant on time (Fig. 4), space (Fig. 5) and energy deposed by the laser :

$$
P_{a b}=g(t) \cdot f(r) \cdot h(E)
$$

with $\mathrm{h}(\mathrm{E})$ deduced from the law of Grün [9] :

$$
h(E)=P_{\text {ref }} \cdot{\frac{E}{E_{\text {ref }}}}^{0.8} \text { where }\left\{\begin{array}{l}
P_{\text {ref }}=5.28 \mathrm{GPa} \\
E_{\text {ref }}=20.6 \mathrm{~J}
\end{array}\right.
$$

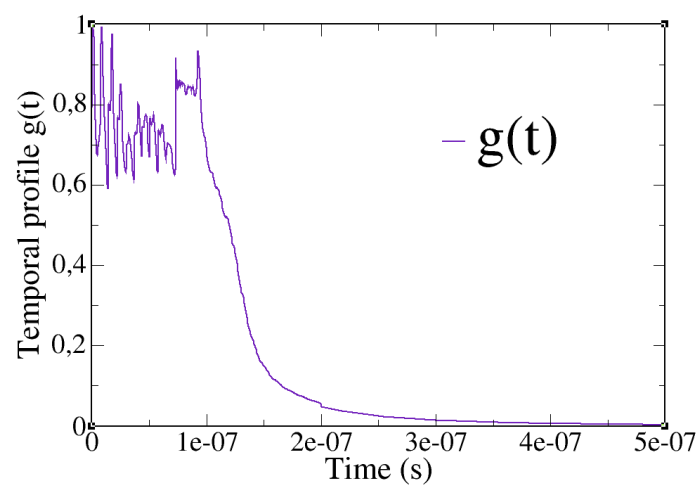

Fig. 4. Normalized temporal profile. Laser configuration : $\varnothing=$ $250 \mu \mathrm{m}, \Delta t=100 \mathrm{~ns}$.

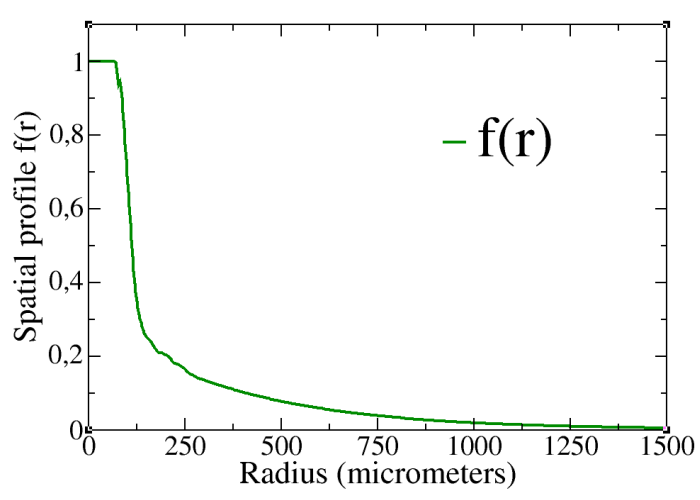

Fig. 5. Normalized spatial profile. Laser configuration : $\varnothing=250$ $\mu m, \Delta t=100 n s$. 


\section{Simulations results and comparison to experiments}

Simplifications of the composite geometry have been realized by neglecting matrix interfaces between the constituants (Fig. 6). Furthermore, thickness af all the constituants are taken equal to their theoretical value. It contrasts with real samples where constituants at surface are thiner due to manufacturing and predamage. Finally, pressure loading is perfectly centered on the Z-fiber. In the further simulations, mesh size of $6.25 \mu \mathrm{m}$ verifies calcul convergence.

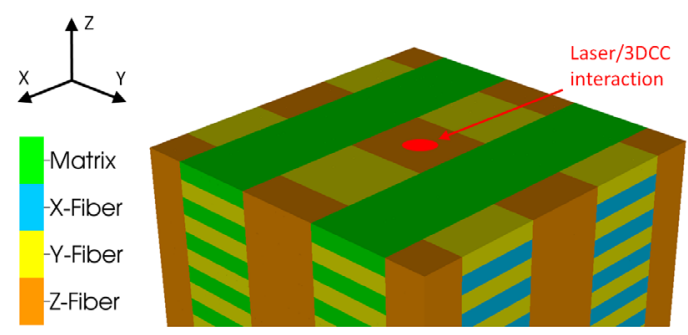

Fig. 6. Simplified model geometry.

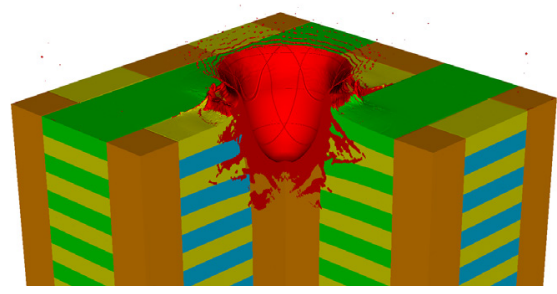

Fig. 7. 3D eulerian simulation corresponding to laser configuration : $\varnothing=250 \mu m, \Delta t=100 n s, E=36 \mathrm{~J}$. Damage material $(\mathrm{D}=1)$ appears in red before.

Simulations using the previously exposed set of parameters led to craters as the one in Fig. 7. In accordance with experimental observations, damage follows interfaces as only targeted Z-fiber and the adjacent mesoconstituant are concerned. It appears that the zone where the parameter $\mathrm{D}=1$ encompass all the experimental crater profile (Fig. 8) with $\mathrm{D}=1$ corresponding to a damaged state. The damage variable used in the model is quite simple as it only considers two possible states. Therefore, it cannot take into account all the phenomena happening during the shock. Due to the microstructure of the material, the predamage state and the complexity of the mesoscale structure, damaged material is not totally pulverised nor intact and a precise meaning of damage is not obvious. Still, we got a model that overall reproduces the totality of the experimental results on a laser energy domain from $13.8 \mathrm{~J}$ to $36 \mathrm{~J}$. Actually, area where $\mathrm{D}=1$ furnish lower and upper limit of the experimental crater. However, difficulties were met to get a good estimation of the crater diameter at surface as initial state surface and predamage significantly influence the results. Nevertheless, damage of the matrix under the surface could explain crater expansion far from the center as this matrix does not retain components above and facilitates debonding.
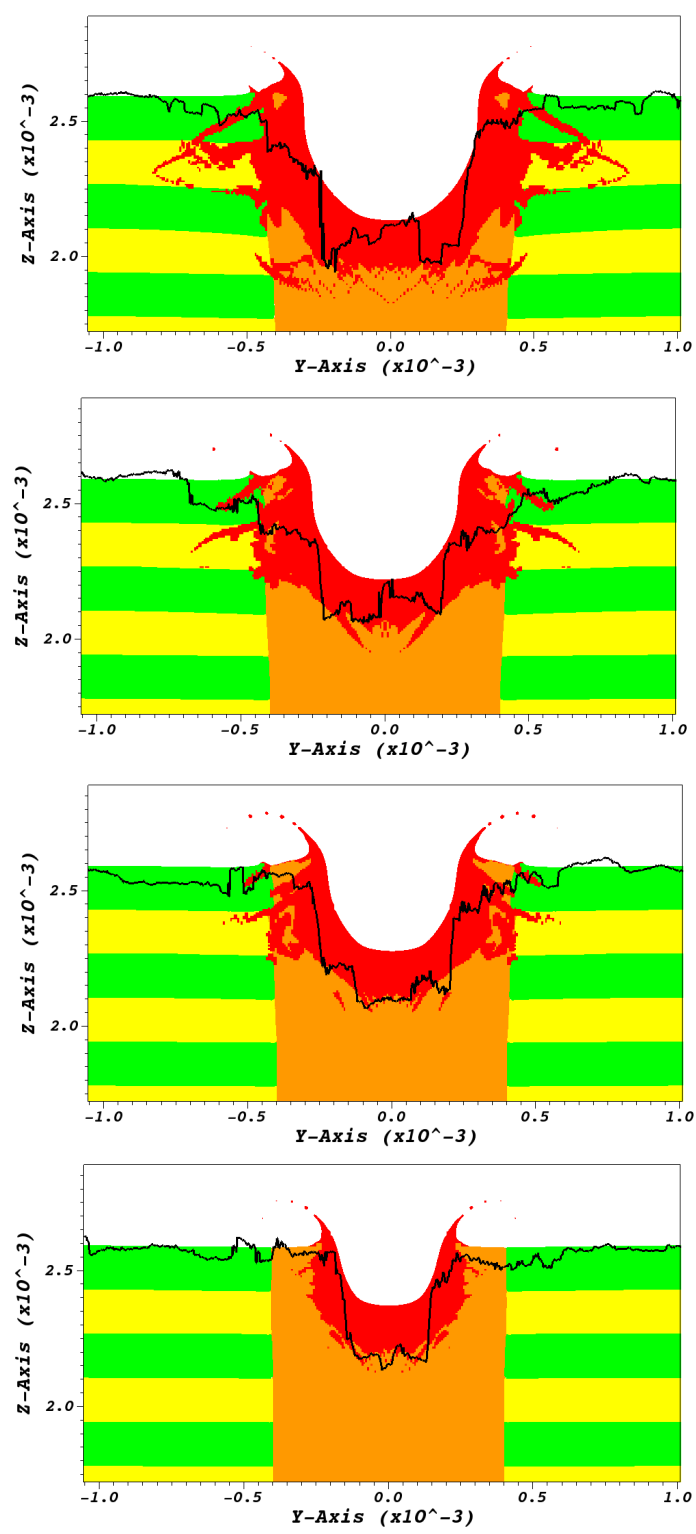

Fig. 8. Comparison of numerical and experimental crater profiles. Simulated damaged material $(\mathrm{D}=1)$ appears in red. The black line represents a slice of the experimental crater. Laser energies for simulation/experiments are respectively (36 J/35.56 $J),(26.3 J / 26.89 J),(20.6 J / 19.64 J)$ and $(13.8 J / 14.01 J)$.

\section{Conclusion}

Laser-induced cratering was performed on a 3D mesoscopic composite. Study of crater profilometric measurements highlighted specificities of the composite damaging under dynamic loading. Those results allowed to calibrate damage coefficients of a mesoscopic numerical model. In spite of the simplifications and imperfections, the model allowed a good estimation of all the experimental crater shapes with the evolution of the laser energy. Therefore, 
mesoscopic model appears as a reliable tool to predict damage in composite under dynamic sollicitation.

\section{References}

1. R.C. Tennyson, C. Lamontagne, Composites: Part A 31, 785-794 (2000)

2. D. Numata, K. Othani, M. Anyoji, K. Takayama, K. Togami, M. Sun, International Journal of Impact Engineering 35, 1695-1701 (2008)

3. M.Gratton, Comportement d'un matériau composite 3D Carb/Carb : Méso-modélisation pour la prévision de la réponse sous choc, Ecole Normale Supérieure de Cachan (1998)
4. J. Sen Gupta, O. Allix, P.-A. Boucard, A. Fanget, P.-L. Héreil, Composites Sciences and Technology 65, 375386 (2003)

5. B. Aubert et al., Presented at 22nd DYMAT Technical Meeting (2016)

6. G. Seisson et al., International Journal of Impact Engineering 63, 18-28 (2014)

7. Gordan R. Johnson, Tim J. Holmquist, AIP Conference Proceeding 309, 981 (1994)

8. B. Aubert, D. Hébert, J.-L. Rullier, L. Videau and L. Berthe, Presented at APS SCCM (2017)

9. J. Grun, R. Decoste, B.H. Hipin and J.Gardner, Applied Physics Letters 39, 545 (1981) 
